\title{
Commentary: Is Sustainability an Option While Implementing Programs to end TB by 2030?
}

\author{
Colleen Longacre', Hala Jassim AIMossawi ${ }^{1}$, Yogan Pillay' ${ }^{2}$, Neeraj Kak ${ }^{1 *}$ \\ 'University Research Co., LLC (URC); Chevy Chase, MD, USA \\ ${ }^{2}$ National Department of Health; Pretoria, Gauteng, South Africa
}

\section{Article Info}

\section{Article Notes}

Received: June 20, 2018

Accepted: July 18, 2019

*Correspondence:

Dr. Neeraj Kak, University Research Co., LLC (URC); Chevy Chase, MD, USA; Email: NKAK@URC-CHS.COM.

(c) 2019 Kak N. This article is distributed under the terms of the Creative Commons Attribution 4.0 International License.
Over the past year, the global tuberculosis (TB) community has rallied around the laudable and ambitious goal of ending TB within a generation. In September 2018, the United Nations General Assembly (UNGA) held its first ever High-Level Meeting (UNHLM) on TB. The resulting UN resolution ${ }^{1}$ reaffirmed its commitment to end the global TB epidemic by 2030 in line with the Sustainable Development Goals (SDGs) target. ${ }^{2}$ Drawing on the global efforts to end TB, including United States Agency for International Development (USAID)'s Global Accelerator to End TB and support from the Global Fund, excellent opportunities for joint and synergistic efforts are possible, including expansion of universal health coverage (UHC), engaging the private sector and civil societies, ensuring equity and respect for human rights, establishment of an innovative approach to strategic information within and beyond health, strengthening of health systems with integrated service delivery, pursuit of sustainable financing, and promotion of research across the spectrum, among others. Published to coincide with World TB Day 2019, the Lancet Commission report on $\mathrm{TB}^{3}$ provides a roadmap for achieving a world free of TB by 2045. However, to achieve this goal, both reports acknowledge the need for a massive increase in financial investment. The UNHLM report calls for global investment for TB to increase from its current level of \$6-7 billion USD to $\$ 13$ billion USD by 2022, while the Lancet Commission report estimates that an additional expenditure of $\$ 5$ billion USD annually over the next two decades is needed.

Countries with high levels of TB and development partners need to seriously consider how to respond to investment of this magnitude within a short timeframe, particularly when it comes to our notion of TB program sustainability. The Commission argues that nearly all high-burden countries can substantially increase domestic funding for $\mathrm{TB}$, and that international donor financing can be targeted to catalyze increases in domestic investment from both the public and private sectors. This emphasis on sustainable financing and donor investment-as-catalyst is also evident in the Global Fund's support for new models of financing ${ }^{4}$ as well as the USAID Global Accelerator to End TB financing strategy. ${ }^{5}$ However, in the context of a TB elimination framework, we cannot only speak about sustainability, we must also talk about the need for widespread escalation and acceleration - if we don't pay now we will pay much more later.

While its operational definition has always been murky, ТВ program sustainability has traditionally focused on maintaining 
program gains while gradually replacing donor-funded investment and capacity with local investment and capacity. If we take the UNHLM commitments seriously, this traditional approach is hugely inadequate to respond to the global targets. Development partners need to rethink their goals and realign their priorities when it comes to TB program sustainability. TB must be given a higher health priority by ironically investing to strengthen the general health systems to improve the performance of TB program along with other health services. In investing in TB programs to strengthen the general health system, it is critical to:

- estimate the resources required and available, and identifying funding gaps in the medium-term;

- ensure that there are sufficient domestic and external resources to achieve the national and global goals;

- ensure patient-centered care where all patients have access to the universal standard of care,

- mobilize societies against TB;

- foster collaboration between TB and HIV programs; and

- integrate TB control in the mainstream of development plans including general health and social services.

Ensuring that we meet the SDG goals in the context of sustainable programming requires a number of key interventions. First, we must address the notion that sustainability efforts means tapering off donor financing for TB. While the Lancet Commission report makes a strong case that nearly all high-burden countries can massively increase domestic annual TB expenditure we must acknowledge the current financing landscape: lowincome countries domestically finance less than $50 \%$ of their TB expenditure, resulting in substantial funding gaps in many of these countries. Also, as new technologies become available for diagnosing and treating TB cases, overall programmatic costs will increase. For example, treating MDR-TB with Bedaquiline and Delaminid increases costs dramatically with the upside being better treatment outcomes. It is unrealistic to expect these countries to both cover these existing funding gaps and accelerate levels of funding under the timeframes needed to meet the UNHLM targets. ${ }^{6}$ Efforts focused on "unlocking" sources of potential domestic spending for TB may be better spent on mobilizing much greater amounts of funding from high-income donor countries. This is particularly true in countries with undemocratic or otherwise challenging political situations, where demonstrated cost-effectiveness and return-on-investment arguments are less likely to yield substantial increases in domestic investment.
Second, we must reconsider the time horizons on which we evaluate TB program sustainability. Traditional thinking on sustainability also suffers from the limited timelines imposed by many donor-funded initiatives. Approaches to sustainability may be better thought of in two phases: 1) pre-TB elimination and 2) post-TB elimination. While we remain in the pre-elimination phase (which the Lancet Commission report estimates we will be until at least 2045), emphasis should remain on increasing investment from all sources. Development partner efforts on sustainability should focus on the effective management and mobilization of this investment. The Lancet Commission report refers to this as the absorption capacity of National TB Programs (NTPs). Development partners can assist NTPs to strengthen their absorption capacity in the following ways (Table 1):

1. Establish effective financial management systems: Development partners can assist NTPs to establish mechanisms by which allocated funds are disbursed, equitably, efficiently and transparently. This could include assisting with: 1) development of TB budget projections; 2) coordination of funding streams to avoid funding gaps and/or duplication of efforts; 3) development of contracting mechanisms for establishment of public-private partnerships and partnerships with other not-for-profit and civil society organizations; and 4) development and implementation of an accountability framework, whereby additional funding disbursements are predicated upon meeting agreed-upon targets.

2. Identify funding priorities: Development partners can assist NTPs to identify priority areas for increased funding and evidence-based interventions where funding would have the greatest impact. This may include: 1) prioritization of NTP goals in context of the country's localized epidemic; 2) development of an operations/implementation research agenda to answer priority questions and to scale effective approaches; 3) use of health systems data to plan interventions and monitor their effectiveness; 4) development of frameworks through which new research findings can be evaluated and effectively translated into new policies and programmatic strategies; 5) leveraging of digital technologies to improve the efficiency and effectiveness of existing TB surveillance and control strategies.

3. Mobilize sufficient human resources and infrastructure: Development partners can assist NTPs to ensure that the increased demand placed on them by the UNHLM targets is met with increased human resource capacity. This may include: 1) forging multi-sectoral partnerships; 2) advocating for the position of the NTP within government structures; 3 ) building the technical and management capacity of NTP leaders; 4) engaging 
Table 1: Summary of Strategies to Strengthen the Absorption Capacity of National TB Programs.

\begin{tabular}{|c|c|}
\hline Strategy & Key Activities \\
\hline \multirow{4}{*}{$\begin{array}{l}\text { Establish effective financial management } \\
\text { systems }\end{array}$} & Develop TB budget projections \\
\hline & Coordinate funding streams to avoid gaps and/or duplication of efforts \\
\hline & $\begin{array}{l}\text { Develop of contracting mechanisms to establish public-private partnerships and partnerships } \\
\text { with civil society organizations }\end{array}$ \\
\hline & Develop and implement an accountability framework \\
\hline \multirow{5}{*}{ Identify funding priorities } & Prioritize NTP goals in the context of the country's localized epidemic \\
\hline & Develop an operations/implementation research agenda \\
\hline & Use health systems data to plan interventions and monitor effectiveness \\
\hline & Develop frameworks to translate new research findings into new policies and strategies \\
\hline & $\begin{array}{l}\text { Leverage digital technologies to improve TB surveillance and control efficiency and effective- } \\
\text { ness }\end{array}$ \\
\hline \multirow{5}{*}{$\begin{array}{l}\text { Mobilize sufficient human resources and } \\
\text { infrastructure }\end{array}$} & Forge multi-sectoral partnerships \\
\hline & Advocate for the position of the NTP within government structures \\
\hline & Build NTP technical and management capacity \\
\hline & Engage NTPs in human resource planning \\
\hline & Build transnational partnerships and research collaboratives \\
\hline \multirow{3}{*}{ Engage the private sector } & Develop accountability mechanisms and results monitoring of private sector providers \\
\hline & Subsidize quality TB diagnostics \\
\hline & Provide adherence support mechanisms to private sector providers \\
\hline \multirow{5}{*}{$\begin{array}{l}\text { Manage the transition from pre-elimination } \\
\text { to post-elimination }\end{array}$} & Manage transition funding mechanisms \\
\hline & Retrain existing personnel on new priorities \\
\hline & Ensure ongoing TB surveillance \\
\hline & Foster cross-border collaborations \\
\hline & Continue TB advocacy and stigma reduction \\
\hline
\end{tabular}

NTPs in human resource planning to ensure adequate capacity and personnel retention at all levels of the health system; and 5) build transnational partnerships and research collaboratives so that effective strategies can be efficiently adopted. Enhanced human resource capacity must also be met with adequate infrastructure investment - in diagnostics, laboratories, supply chains, and pharmaceutical management.

4. Engage the private sector: The Lancet Commission Report points out that untreated and poorly treated patients who seek care in the private sector are a major source of ongoing TB transmission. Of the 3.6 million missing TB patients (those whose cases were not notified to TB programs), more than half (56\%) were from seven countries where primary care is primarily provided by the private sector (India, Indonesia, Nigeria, Philippines, Pakistan, Bangladesh, and Myanmar). Improving the diagnosis and treatment of these private sector patients through integrated service delivery models is key to optimizing patient outcomes and sustaining ongoing TB elimination efforts. However, while this engagement with the private sector has the potential to greatly expand access to high quality care, such engagement costs money and will require additional investment by NTPs and development partners to forge and sustain these private sector linkages. A modeling study commissioned by the Lancet Commission found that optimal private sector engagement could avert 8 million TB deaths in India over the next 30 years, but that it would require an annual increase of $\$ 290$ million in national TB program costs. Development partners can assist NTPs in building the sustainability of their private sector engagement through the development of mechanisms of accountability and results monitoring for private sector providers, subsidization of quality TB diagnostics, and adherence support mechanisms in the private sector.

5. Manage the transition from pre-elimination to postelimination: As countries move closer to the UNHLM targets, the nature of their TB epidemics and the strategies for addressing it will necessarily change. Development partners can assist NTPs in this long-term strategic planning, including: 1) managing transitioning funding mechanisms; 2) retraining existing personnel on new priority areas and interventions; 3 ) ensuring ongoing TB surveillance to maintain achieved levels of TB control; 4) fostering cross-border collaborations to minimize the risk of ongoing TB spread; and 5) continuing TB advocacy and stigma reduction activities so that TB does not remain in pockets of vulnerable populations that could undermine global control efforts.

The Lancet Commission report is ambitious in its scope and optimistic in its assertion that a TB-free world is possible within a generation. Meeting this 
highly worthy challenge will require that development partners rethink their traditional notions of TB program sustainability. Rather than focusing on transitioning away from donor financing, development partner efforts toward sustainability should focus on two main goals: 1) massively increasing global investment in the short to medium term; and, 2) increasing the absorption capacity of high-burden countries to effectively translate this investment into program success.

\section{References}

1. A/RES/73/3. Political declaration of the high-level meeting of the General Assembly on the fight against tuberculosis. https://www. un.org/en/ga/search/view_doc.asp?symbol=A/RES/73/3. Published 2018.
2. Official Records of the UN General Assembly. The 2030 Agenda for Sustainable Development, A/RES/70/1. 2015; 16301(October): 13-14.

3. Reid MJA, Arinaminpathy N, Bloom A, et al. Building a tuberculosisfree world: The Lancet Commission on tuberculosis. Lancet (London, England). 2019; 393(10178): 1331-1384. doi:10.1016/S01406736(19)30024-8

4. The Global Fund To Fight AIDS, TB and Malaria. The Global Fund New Funding Model. 2016.

5. The United States Agency for International Development. USAID Global Accelerator to End TB. https://www.usaid.gov/what-we-do/ global-health/tuberculosis/resources/news-and-updates/globalaccelerator-end-tb. Published 2019. Accessed May 29, 2019.

6. Chang AY, Cowling K, Micah AE, et al. Past, present, and future of global health financing: a review of development assistance, government, out-of-pocket, and other private spending on health for 195 countries, 1995-2050. Lancet. 2019. doi:10.1016/S0140-6736(19)30841-4 